\title{
Discrete Symmetry Tests In Neutron-induced Compound States
}

\author{
H.M.Shimizu*1, W.M.Snow ${ }^{2}$, V.P.Gudkov ${ }^{3}$, J.D.Bowman ${ }^{4}$, J.Curole ${ }^{2}$, B.M.Goodson ${ }^{5}$,

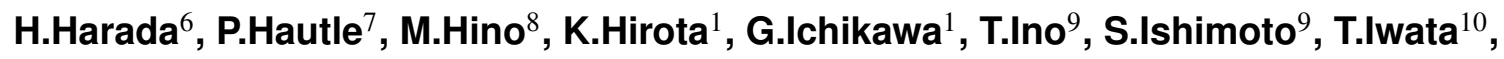 \\ A.Kimura ${ }^{6}$, M.Kitaguchi ${ }^{1}$, Y.Kiyanagi ${ }^{1}$, J.Koga ${ }^{11}$, K.Mishima ${ }^{9}$, Y.Miyachi ${ }^{5}$, \\ T.Momose $^{12}$, T.Morishima ${ }^{1}$, K.Nagamoto ${ }^{1}$, A.Okada ${ }^{1}$, T.Okudaira ${ }^{1}$, S.Penttila ${ }^{4}$, \\ B.Plaster $^{13}$, K.Sakai ${ }^{6}$, D.Schaper ${ }^{13}$, S.Takada ${ }^{11}$, K.Taketani ${ }^{9}$, X.Tong ${ }^{4}$, A.S.Tremsin ${ }^{14}$, \\ Y.Yamagata $^{15}$, T.Yamamoto ${ }^{1}$, N.Yamanaka ${ }^{15}$, M.Yokohashi ${ }^{1}$, T.Yoshioka ${ }^{2}$ \\ ${ }^{1}$ Nagoya University, Furocho, Chikusa, Nagoya 464-8602, Japan \\ ${ }^{2}$ Indiana University, Bloomington, Indiana 47408, U.S.A. \\ ${ }^{3}$ University of South Carolina, Columbia, South Carolina 29208, U.S.A. \\ ${ }^{4}$ Oak Ridge National Laboratory, Oak Ridge, Tennessee 37831, U.S.A. \\ ${ }^{5}$ Southern Illiois University, 1245 Lincoln Drive, Carbondale, Illinois, 62901, U.S.A. \\ ${ }^{6}$ Japan Atomic Energy Agency, 2-4 Shirakata, Tokai, Ibaraki 319-1195, Japan \\ ${ }^{7}$ Paul Scherrer Institut, Villigen, $\mathrm{CH}-5232$, Switzerland \\ ${ }^{8}$ Research Reactor Institute, Kyoto University, Kumatori, Osaka 590-0494, Japan \\ ${ }^{9}$ High Energy Accelerator Research Organization (KEK), 1-1 Oho, Tsukuba 305-0801, Japan \\ ${ }^{10}$ Yamagata University, 1-41-2 Koshirakawa, Yamagata 990-8560, Japan \\ ${ }^{11}$ Kyushu University, 744 Motooka, Nishi, Fukuoka 819-0395, Japan \\ ${ }^{12}$ University of British Columbia, Vancouver, British Columbia V6R1Z1, Canada \\ ${ }^{13}$ Kentucky University, Lexington, Kentucky 40506, U.S.A. \\ ${ }^{14}$ University of California at Berkeley, 7 Gauss Way, Berkeley, California 94720, U.S.A. \\ ${ }^{15}$ RIKEN, 2-1 Hirosawa, Wako, Saitama 351-0198, Japan \\ E-mail: shimizudphi.phys.nagoya-u.ac.jp
}

A possibility of new type experimental searches for new physics beyond the standard model is being introduced in the symmetry tests in neutron-induced compound states according to the successful operation of intense pulsed neutron sources. The basis of experiment design is discussed.

The 26th International Nuclear Physics Conference

11-16 September, 2016

Adelaide, Australia

* Speaker. 


\section{Introduction}

One of the motivations to search for new physics beyond the standard model in the CPviolating observables is to eliminate the large discrepancy of the baryon asymmetry of the universe in the observation of the cosmic microwave background and that in the theoretical estimation based on the standard model. The electric dipole moment of neutrons is a T-violating observable which signals the breaking of CP-symmetry via the CPT-theorem. Continuous efforts have been paid to improve the experimental sensitivity to the electric dipole moment of neutrons and new experimental challenges are in progress [四].

Another possibility of the high-sensitivity search for the T-violation has been discussed [ם, [3] after the discovery of extremely large parity-violation effects in neutron-induced compound states [四, []]. In the case the forward scattering amplitude of neutrons can be described in the form

$$
f=A+B \boldsymbol{\sigma} \cdot \hat{\boldsymbol{I}}+C \boldsymbol{\sigma} \cdot \hat{\boldsymbol{k}}+D \boldsymbol{\sigma} \cdot(\hat{\boldsymbol{I}} \times \hat{\boldsymbol{k}})
$$

T-violation can be searched for in the $D$-term, where $\boldsymbol{\sigma}, \hat{\boldsymbol{k}}$ and $\hat{\boldsymbol{I}}$ are unit vectors of the neutron spin, the neutron momentum and the target nuclear spin [目]. This type of experiments require an intense epithermal neutron beam and a spin-polarized nuclear targets. The successful operation of the intense spallation neutron sources of the Japan Proton Accelerator Research Complex (J-PARC) and the Oak Ridge National Laboratory has enabled the opportunity to carry out the T-violation searches in neutron-induced compound states. The spin polarized nuclear target is the missing component. The large nuclear polarization requires intensive technical efforts and its method depends on nucleus to be polarized. In this paper, we describe the study of the interference between partial waves of incident neutrons in the entrance channel to the compound states for the selection of appropriate target nuclei.

\section{Parity Violation in Compound States}

The magnitude of the parity violating effects in the effective nucleon-nucleon interaction is at the level of $10^{-7}$ as observed in the helicity dependence of the total cross section between nucleons $[\mathbb{Q}, \mathbb{8}, \mathbf{Q}]$. Extremely large parity violation was found in the helicity dependence of the absorption cross section of epithermal neutrons at p-wave resonances of ${ }^{139} \mathrm{La}$ [团]. The large parity violation was explained as the interference between the amplitudes of the p-wave resonance and a neighboring s-wave resonance [ए]]. The ratio of the helicity dependent cross section to the p-wave resonance cross section is referred to as the longitudinal asymmetry denoted as $A_{\mathrm{L}}$. The longitudinal asymmetry was intensively studied in neutron transmission and in $(n, \gamma)$ measurements [[]]. The $\gamma$-ray energy dependence with the moderate energy resolution was not found in $(n, \gamma)$ measurements, which implies that the interference is taking place in the entrance channel to the compound state and not in the exit channel [W]].

The spin of the compound state $\boldsymbol{J}$ is given as

$$
\boldsymbol{J}=\boldsymbol{I}+\boldsymbol{s}+\boldsymbol{l}
$$


where $\boldsymbol{I}$ is the target nuclear spin and $\boldsymbol{s}$ and $\boldsymbol{l}$ are the spin and orbital angular momentum of incident neutrons. The total angular momentum of incident neutron is given as

$$
\boldsymbol{j}=\boldsymbol{s}+\boldsymbol{l}
$$

and the channel spin in the entrance channel as

$$
\boldsymbol{S}=\boldsymbol{I}+\boldsymbol{s}
$$

We put the neutron width of the p-wave resonance as $\Gamma_{\mathrm{p}}^{\mathrm{n}}$ and that of the neighboring s-wave resonance as $\Gamma_{\mathrm{s}}^{\mathrm{n}}$. The p-wave incident neutron has two components in terms of the total angular momentum of incident neutron of $j=1 / 2$ and $j=3 / 2$. We put corresponding partial neutron widths as $\Gamma_{\mathrm{p}}^{\mathrm{n}}\left(j=\frac{1}{2}\right)$ and $\Gamma_{\mathrm{p}}^{\mathrm{n}}\left(j=\frac{3}{2}\right)$. If we write

$$
x^{2}=\frac{\Gamma_{\mathrm{p}}^{\mathrm{n}}\left(j=\frac{1}{2}\right)}{\Gamma_{\mathrm{p}}^{\mathrm{n}}}, \quad y^{2}=\frac{\Gamma_{\mathrm{p}}^{\mathrm{n}}\left(j=\frac{3}{2}\right)}{\Gamma_{\mathrm{p}}^{\mathrm{n}}},
$$

the $x$ and $y$ satisfies the relation $x^{2}+y^{2}=1$. We put $x=\cos \phi$ and $y=\sin \phi$ and we refer to $\phi$ as the mixing angle between the two components corresponding to $j=1 / 2$ and $j=3 / 2$. The longitudinal asymmetry $A_{\mathrm{L}}$ is given as

$$
A_{\mathrm{L}}=-\frac{2 x W}{\left|E_{\mathrm{p}}-E_{\mathrm{s}}\right|} \sqrt{\frac{\Gamma_{\mathrm{s}}^{\mathrm{n}}}{\Gamma_{\mathrm{p}}^{\mathrm{n}}}}
$$

within the framework of the interference between an s-wave and a p-wave resonances, where $E_{\mathrm{p}}$ and $E_{\mathrm{s}}$ are the resonance energies of the p-wave and s-wave resoances and $W$ the weak matrix element. The experimental values of $\left|A_{\mathrm{L}}\right|$ is summarized in Figure $\mathrm{W}$. They are obviously beyond the typical P-violatiing effects in the effective nucleon-nucleon interaction. The universality of Eq. 2.5 over P-violating nuclei was ensured in the likelihood analysis of the matrix element $W$ taking the $x$ as a variable since the values of $x$ were not known.

\section{Time Reversal Invariance Violation in Compound States}

The T-violating effect is estimated as

$$
\Delta \sigma_{\mathrm{T}}=\kappa(J) \frac{W_{\mathrm{T}}}{W} \Delta \sigma_{\mathrm{P}}
$$

where $\Delta \sigma_{\mathrm{P}}$ and $\Delta \sigma_{\mathrm{T}}$ are $\mathrm{P}$-violating and T-violating cross sections, $W_{\mathrm{T}}$ is the T-violating matrix element [వ]. The $\kappa(J)$ is the geometric factor to connect two representations of different coupling sequence of angular momenta: $\boldsymbol{J}=\boldsymbol{I}+\boldsymbol{j}=\boldsymbol{S}+\boldsymbol{l}$. Two channel spin components $S=I+1 / 2$ and $S=I-1 / 2$ contribute to the T-violating effects. We define their partial neutron widths as $\Gamma_{\mathrm{p}}^{\mathrm{n}}(S=I+1 / 2)$ and $\Gamma_{\mathrm{p}}^{\mathrm{n}}(S=I-1 / 2)$ and put

$$
x_{s}^{2}=\frac{\Gamma_{\mathrm{p}}^{\mathrm{n}}(S=I+1 / 2)}{\Gamma_{\mathrm{p}}^{\mathrm{n}}}, \quad y_{s}^{2}=\frac{\Gamma_{\mathrm{p}}^{\mathrm{n}}(S=I-1 / 2)}{\Gamma_{\mathrm{p}}^{\mathrm{n}}} .
$$




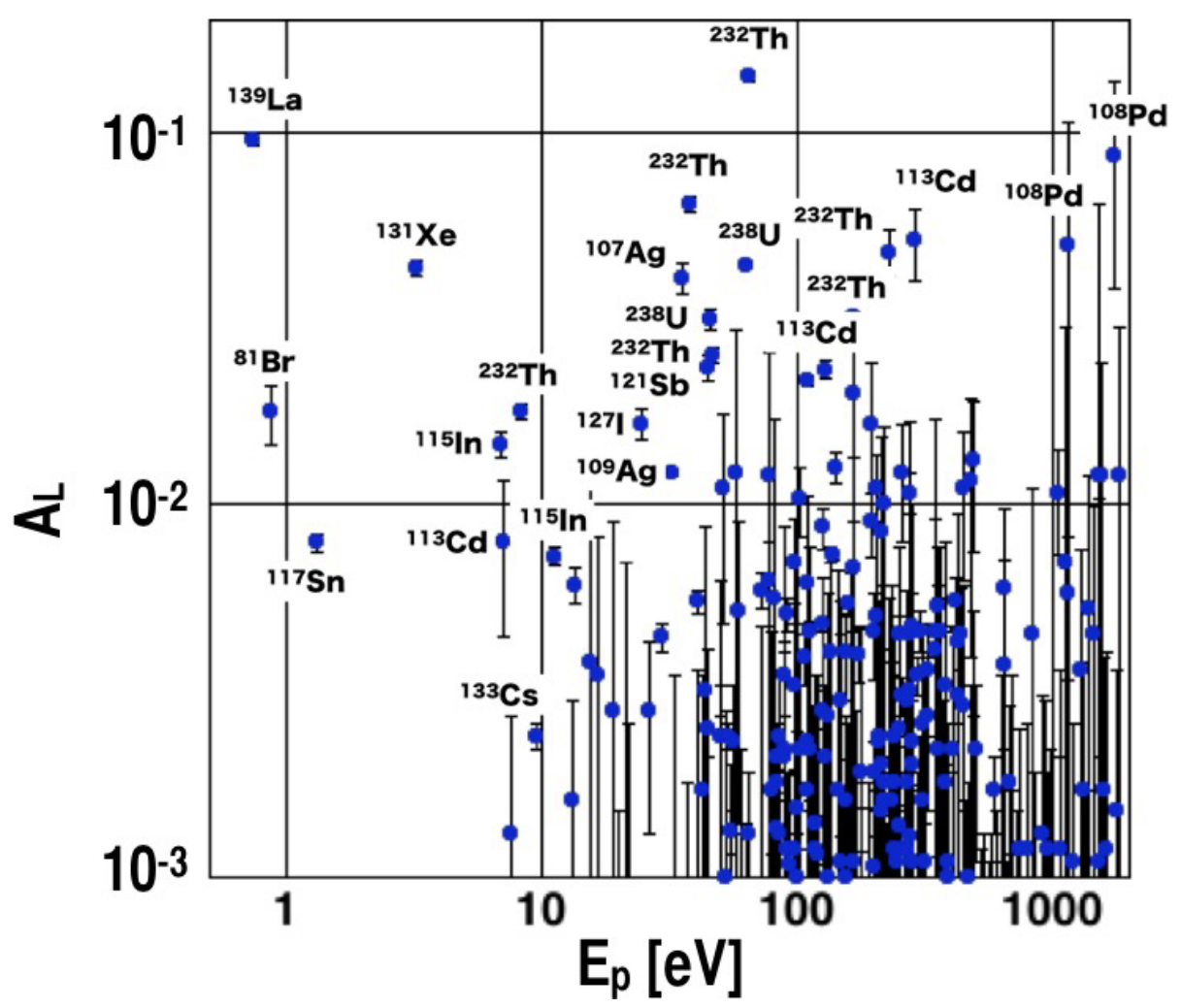

Figure 1: Experimental values of $\left|A_{\mathrm{L}}\right|$ summarized in Ref. [目] are plotted. The horizontal axis is the resonance energy of corresponding $\mathrm{p}$-wave resonance.

The $x_{s}$ and $y_{s}$ are related to $x$ and $y$ through the relation

$$
\begin{aligned}
& x_{s}=(-1)^{2 I+1} \sqrt{4 I}\left\{\begin{array}{ccc}
1 & \frac{1}{2} & \frac{1}{2} \\
I & J & I-\frac{1}{2}
\end{array}\right\} x+(-1)^{2 I} \sqrt{8 I}\left\{\begin{array}{ccc}
1 & \frac{1}{2} & \frac{3}{2} \\
I & J & I-\frac{1}{2}
\end{array}\right\} y \\
& y_{s}=(-1)^{2 I} \sqrt{4(I+1)}\left\{\begin{array}{ccc}
1 & \frac{1}{2} & \frac{1}{2} \\
I & J & I+\frac{1}{2}
\end{array}\right\} x+(-1)^{2 I+1} \sqrt{8(I+1)}\left\{\begin{array}{ccc}
1 & \frac{1}{2} & \frac{3}{2} \\
I & J & I+\frac{1}{2}
\end{array}\right\} y .
\end{aligned}
$$

The explicit expression of $\kappa(J)$ is given in Ref. [D] as the function of $x_{s}$ and $y_{s}$ for cases of $J=$ $I+1 / 2$ and $J=I-1 / 2$ as

$$
\begin{aligned}
& \kappa\left(J=I+\frac{1}{2}\right)=-\frac{3}{2 \sqrt{2}}\left(\frac{2 I+1}{2 I+3}\right)^{3 / 2}\left(\frac{3}{\sqrt{2 I+3}} \frac{x_{s}}{y_{s}}-\sqrt{I}\right)^{-1} \\
& \kappa\left(J=I-\frac{1}{2}\right)=-\frac{3}{2 \sqrt{2}}\left(\frac{2 I+1}{2 I-1}\right)\left(\frac{I}{I+1}\right)^{1 / 2}\left(-\frac{I-1}{\sqrt{2 I-1}} \frac{y_{s}}{x_{s}}+\sqrt{I+1}\right)^{-1} .
\end{aligned}
$$

This relation can be expressed with $x$ and $y$ by using Eq. 3.4 as

$$
\begin{aligned}
& \kappa\left(J=I+\frac{1}{2}\right)=\frac{3}{2 \sqrt{2}} \frac{(2 I+1)^{3 / 2}}{2 I+3} \frac{2 \sqrt{I} x-\sqrt{2 I+3} y}{(2 I-3) \sqrt{2 I+3} x-(2 I+9) \sqrt{I} y}, \\
& \kappa\left(J=I-\frac{1}{2}\right)=-\frac{3}{2 \sqrt{2}} \frac{(2 I+1) \sqrt{I}}{\sqrt{(I+1)(2 I-1)}} \frac{2 \sqrt{I+1} x+\sqrt{2 I-1} y}{(I+3) \sqrt{2 I-1} x-(5 I-3) \sqrt{I+1} y} .
\end{aligned}
$$


These equations visualizes that the T-violation sensitivity strongly depends on the values of $x$ and $y$ or $\phi$ the mixing angle of $j=1 / 2$ and $j=3 / 2$ components.

\section{Experiment Design}

The sensitivity to new physics of the T-violating effects in compound states is not easily compared with that of the neutron electric dipole moment. A theoretical analysis is suggesting a crude estimation within the framework of an effective field theory [미]. The suggested sensitivity crudely equivalent to the present upper limit of the neutron electric dipole moment corresponds to the submillibarn accuracy in terms of the cross section determination accuracy, which can be accessed within a day using the intense spallation neutron source. The p-wave resonances in low energy regions are more appropriate since the neutron intensity of the spallation neutron sources such as that of the Material and Life-science Facility of the Japan Proton Accelerator Research Complex (J-PARC/MLF) rapidly decrease in higher energies. Target nuclear polarization is necessary for Tviolation. Thus, we are surveying the candidate nuclei among non-zero-spin nuclei showing large P-violating effects in lower energy resonance energy: ${ }^{139} \mathrm{La},{ }^{81} \mathrm{Br},{ }^{131} \mathrm{Xe},{ }^{117} \mathrm{Sn},{ }^{115} \mathrm{In}, \ldots$

However, the information of the mixing angle $\phi$ is necessary to quantify the sensitivity to Tviolation. The interference of $j=1 / 2$ and $j=3 / 2$ components are expected to cause the neutronenergy-dependent angular distribution of individual $\gamma$-rays as suggested in Ref. [ए3]]. The experimental study of the angular distribution in resolved $\gamma$-ray detection is in progress at the ANNRI Spectrometer installed at BL04 of the Material and Life-science Facility of the Japan Proton Accelerator Research Complex to determine the mixing angle $\phi$ [14]]. The practical design of the sensitive search for new physics beyond the standard model will be enabled based on the determination of $\phi$.

\section{References}

[1] B.Lauss et al, in this proceeding.

[2] V.P.Gudkov, Phys. Rep. 212 (1992) 77.

[3] J.D.Bowman and V.Gudkov, Phys. Rev. C 90 (2014) 065503.

[4] V.P.Alfimenkov et al., Nucl. Phys. A 398 (1983) 93.

[5] G.E.Mitchell et al., Phys. Rep. 354 (2001) 157 and references therein.

[6] P.K.Kabir, Phys. Rev. D 37 (1988) 1856.

[7] J.M.Potter et al., Phys. Rev. Lett. 33 (1974) 1307.

[8] V.Yuan et al., Phys. Rev. Lett. 57 (1986) 1680.

[9] E.G.Adelberger and W.C.Haxton, Ann. Rev. Nucl. Part. Sci. 35 (1985) 501.

[10] O.P.Sushkov and V.V.Flambaum, Usp. Fiz. Nauk 136 (1982) 3 (O.P.Sushkov and V.V.Flambaum, Sov. Phys. Uspekhi 25 (1982) 1).

[11] H.M.Shimizu et al., Nucl. Phys. A 552 (1993) 293.

[12] Y.-H.Song et al., Phys. Rev. C83 (2011) 065503. 
[13] V.V.Flambaum, Nucl. Phys. A 435 (1985) 352.

[14] T.Okudaira et al, in this proceeding. 